Urologe 2017·56:917-924

DOI 10.1007/s00120-017-0382-8

Online publiziert: 12. April 2017

(c) Der/die Autor(en) 2017. Dieser Artikel ist

eine Open-Access-Publikation.

CrossMark

\author{
D. Anderson ${ }^{1} \cdot$ J. Lehmann ${ }^{2} \cdot$ T. Ecker ${ }^{1} \cdot$ S. Vosgerau ${ }^{3} \cdot$ V. Donatz ${ }^{3}$ \\ ${ }^{1}$ Ecker + Ecker GmbH, Hamburg, Deutschland \\ ${ }^{2}$ Klinik für Urologie, Städtisches Krankenhaus, Kiel, Deutschland \\ ${ }^{3}$ Ferring Arzneimittel GmbH, Kiel, Deutschland
}

\title{
Kosteneffektivität von GnRH- Antagonisten bei Patienten mit Prostatakarzinom und kardiovaskulärem Risiko
}

\section{Vergleichende Analyse gegenüber Leuprorelin anhand der Number Needed to Treat}

\begin{abstract}
Im Rahmen der Androgendeprivationstherapie (ADT) bei Patienten mit fortgeschrittenem Prostatakarzinom (fPCa) wird anhand der "number needed to treat" (NNT) für kardiovaskuläre (KV-)Ereignisse die Kosteneffektivität für GnRHAntagonisten beschrieben.
\end{abstract}

\section{Hintergrund}

Zur gesundheitsökonomischen Bewertung und Vergleich von medizinischen Interventionen werden in der Regel Ergebnisse aus klinischen Studien herangezogen. Dies kann z. B. im Rahmen einer Kosteneffektivitätsanalyse (KEA) geschehen, wobei die medizinischen Outcomes zu den einzusetzenden Kosten in Bezug gesetzt werden und die Ergebnisse verschiedener Therapiealternativen verglichen werden können [3].

Die Task Force zur Verwendung gesundheitsökonomischer Informationen bei Therapieentscheidungen im Gesundheitswesen der internationalen Gesellschaft für Pharmaökonomie und Outcomes Research stellte im Rahmen einer systematischen Literaturrecherche fest, dass die NNT häufig als Entscheidungshilfe von Ärzten verwendet wird und dass die NNT im Rahmen einer KEA Ärzten helfen kann, Ergebnisse aus gesundheitsökonomischen Studien besser $\mathrm{zu}$ verstehen und anzuwenden [11].

Die vorliegende gesundheitsökonomische Evaluation befasst sich unter Verwendung der NNT mit der KEA zweier Hormontherapien, die beim fPCa eingesetzt werden. Als Outcome wird die Verhinderung von KV-Ereignissen oder Tod anhand der NNT herangezogen. Die NNT gibt dabei die Anzahl von Patienten an, die über einen bestimmten Zeitraum eine neue Therapie anstelle einer Standardbehandlung erhalten müssen, um ein KV-Ereignis zu verhindern.

Aktuelle Studien und Übersichtsarbeiten legen nahe, dass unter ADT bei Patienten mit fPCa und einer KV-Vorgeschichte ein erhöhtes Risiko für erneute KV-Ereignisse besteht [6, 20, 30]. Dieses Risiko scheint zwischen den GnRHAgonisten Leuprorelin und Goserelin und -Antagonisten unterschiedlich ausgeprägt, wobei der etwas teurere GnRHAntagonist ein vorteilhafteres Risikoprofil aufweist [2].

\section{Fragestellung}

In dieser Studie wurde untersucht, ob der Einsatz von Degarelix im Vergleich zu Leuprorelin im Rahmen einer ADT-Behandlung von Patienten mit einem fPCa und einer KV-Vorgeschichte kosteneffektiv ist.

\section{Methodik}

Bei der Betrachtung der Kosten wurde die Perspektive der gesetzlichen Krankenversicherung (GKV) eingenommen, da es sich um den relevanten Kostenträger für Leistungen zur Therapie des PCa handelt. Die Betrachtung bezieht sich auf den Zeitraum eines Jahres.

Zur Beantwortung der Fragestellung wurden die Ergebnisse einer gepoolten Analyse von sechs randomisierten und kontrollierten Studien herangezogen, die zum Vergleich der GnRH-Agonisten Leuprorelin und Goserelin mit dem GnRH-Antagonisten Degarelix vorliegen [2]. Dabei wurden ausschließlich KV-Ereignisse oder Tod im Rahmen der Hormontherapie bei Patienten mit fPCa betrachtet.

Die Population der eingeschlossenen Studien (- Tab. 1) umfasst 2328 hormontherapienaive Männer mit ei- 
Tab. 1 Übersicht berücksichtigter Studien

\begin{tabular}{|ll|l}
\hline Studie $^{\text {a }}$ & Dauer (Monate) & Komparator \\
\hline CS21 (NCT00295750) & 12 & Leuprorelin \\
\hline CS35 (NCT00946920) & 12 & Goserelin \\
\hline CS37 (NCT00928434) & 14 & Leuprorelin \\
\hline CS28 (NCT00831233) & 3 & Goserelin \\
\hline CS30 (NCT00833248) & 3 & Goserelin \\
CS31 (NCT00884273) & 3 & Goserelin \\
\hline${ }^{a}$ Es traten in 3 von 6 Studien KV-Ereignisse auf (CS21, CS35, CS37) & \\
\hline
\end{tabular}

\begin{tabular}{lllll}
\hline $\begin{array}{l}\text { Tab. } 2 \\
\text { GnRH }\end{array}$ & KV-Ereignisse nach Albertsen et al. [2] & \multicolumn{2}{l}{ Davon mit KV-Ereignis } \\
& Patienten mit KV-Vorgeschichte & \multicolumn{2}{l}{ (n) } & (\%) \\
\hline Agonist & 245 & 36 & 14,7 \\
\hline Antagonist & 463 & 30 & 6,5
\end{tabular}

Tab. 3 Endpunktoperationalisierung zur Kostenermittlung

\section{Endpunkt}

Myokardinfarkt

ICD-10-2014 [2, 8]

Ischämische zerebrovaskuläre Zustände

Hämorrhagische zerebrovaskuläre Zustände

Arterielle Embolien und thrombotische Ereignisse

Andere ischämische Herzerkrankung

nem histopathologisch bestätigten $\mathrm{PCa}$, einem ECOG-Score <2 und einer Mindestlebenserwartung von 12 Monaten. Patienten mit einer weiteren Krebsdiagnose in den letzten 5 Jahren vor Studieneinschluss wurden nicht eingeschlossen.

In der Studie wurde ein kombinierter Endpunkt aus KV-Ereignissen und Tod jeglicher Ursache betrachtet. KVEreignisse wurden als folgende schwere unerwünschte Ereignisse definiert: Myokardinfarkt, ischämisches zerebrovaskuläres Ereignis, hämorrhagisches zerebrovaskuläres Ereignis, arterielle Embolie und thrombotisches Ereignis, andere ischämische Herzerkrankung.

Des Weiteren wurde die Subgruppe der Patienten analysiert, die bereits ein KV-Ereignis in der Vorgeschichte hatten (•Tab. 2). Die Auswertungen wurden mittels Kaplan-Meier-Kurven und Logrank-Tests durchgeführt, um die Zeit bis zum KV-Ereignis oder zum Tod zwischen den Interventionen zu vergleichen.

Die Analyse ergab, dass bei Männern mit vorbestehender KV-Erkrankung das Risiko von KV-Ereignissen innerhalb des

relix betrug 8,2 Prozentpunkte.

In einem ersten Schritt wurden zunächst die relevanten NNT mittels des Statistikprogramms „R“ nachvollzogen, um durch Angabe der Konfidenzintervalle die Robustheit der NNT zu belegen. Die Berechnungen wurden mit der metaanalytischen Methode der „inverse variance“ mit dem Simonian-Laird Estimator für $\mathrm{Tau}^{2}$ durchgeführt.

Das Ergebnis der gepoolten Analyse $(\mathrm{NNT}=12)$ diente als Grundlage für eine Schätzung der Auswirkungen von Degarelix auf das Budget der GKV („budget impact“). Dabei stellt die Analyse für 12 Patienten die zusätzlichen Arzneimittelkosten der Degarelix-Therapie den Kosten für ein (vermiedenes) KVEreignis gegenüber (•Abb. 1).

Basierend auf dem kombinierten Endpunkt aus KV-Ereignissen oder Tod wurde die Analyse unter der Annahme durchgeführt, dass die Todesfälle auf kardiovaskuläre Ereignisse zurückzuführen sind.

\section{Arzneimittelkosten}

Bei der Auswahl des Vergleichspräparats und der Ermittlung der Arzneimittelkosten wurde auf Angaben im ABDAArtikelstamm und die Ergebnisse einer Analyse von Verordnungsdaten von „IMS Health“ zurückgegriffen. Dabei wurde das verordnungsstärkste Leuprorelin-Präparat als primärer Komparator gewählt. Als Arzneimittelkosten gehen dabei jeweils die GKV-Kosten pro Packung ein, die sich aus dem Apothekenabgabepreis nach Abzug der gesetzlichen Rabatte der pharmazeutischen Hersteller und der Apotheken ergeben ( $\$ \$ 130,130$ a SGB V). Hinzu kommen sich aus den Fachinformationen ergebende Kosten für zusätzlich notwendige Leistungen zu Lasten der GKV im Rahmen der Therapie (zGKVL.). Die relevanten Kosten wurden dabei in Anlehnung an $\$ 4$ Abs. 8 der Arzneimittelnutzenverordnung (AMNutzenV, G-BA) ermittelt. So wurden nur die Leistungen aufgeführt, bei denen es regelhaft Unterschiede zwischen den $\mathrm{zu}$ vergleichenden Interventionen gibt.

\section{Ereigniskosten}

Die Kosten für ein KV-Ereignis wurden aus den folgenden Bereichen ermittelt: (1) Notfallversorgung, (2) Behandlung im Krankenhaus und (3) Rehabilitation.

Für die Ermittlung der Kosten für die Notfallversorgung sind im Rahmen des $₫ 60$ SGB V (Fahrkostenregelung) und der Krankentransport-Richtlinie des Gemeinsamen Bundesausschusses (RLKrankentransport, G-BA) verschiedene Beförderungsmittel möglich. Welches Beförderungsmittel zu verordnen ist, ist anhand der zwingenden medizinischen Notwendigkeit im Einzelfall unter Beachtung des Wirtschaftlichkeitsgebots $\mathrm{zu}$ entscheiden ( $\$ 12$ SGB V). Bei den 
Urologe 2017·56:917-924 DOI 10.1007/s00120-017-0382-8

(c) Der/die Autor(en) 2017. Dieser Artikel ist eine Open-Access-Publikation.

\section{Anderson · J. Lehmann · T. Ecker · S. Vosgerau · V. Donatz}

\section{Kosteneffektivität von GnRH-Antagonisten bei Patienten mit Prostatakarzinom und kardiovaskulärem Risiko. Vergleichende Analyse gegenüber Leuprorelin anhand der Number Needed to Treat}

\section{Zusammenfassung}

Hintergrund. Aktuelle Studien zeigen, dass unter Androgendeprivationstherapie bei hormonsensitiven Prostatakarzinom(PCa-)Patienten mit kardiovaskulärer (KV)Vorgeschichte ein erhöhtes Risiko für erneute KV-Ereignisse besteht. Hierbei weist der etwas teurere GnRH- (Gonadotropin-ReleasingHormon-)Antagonist im Vergleich zu den GnRH-Agonisten Leuprorelin und Goserelin ein vorteilhafteres Risikoprofil auf. Es wurde untersucht, ob eine Behandlung dieser Patienten mit Degarelix im Vergleich zu Leuprorelin kosteneffektiv ist.

Methoden. Diese Evaluation basiert auf einer gepoolten Analyse von sechs randomisiert kontrollierten Phase-III-Studien zum Vergleich von Degarelix und den GnRH-Agonisten
Leuprorelin und Goserelin. Für einen Endpunkt aus KV-Ereignissen oder Tod wurde mit einer „number needed to treat" von 12 eine Überlegenheit von Degarelix festgestellt. Aus Sicht der gesetzlichen Krankenversicherung (GKV) wurden die zusätzlichen DegarelixArzneimittelkosten den Kosten eines vermiedenen KV-Ereignisses gegenüber gestellt. Es wurden Kosten für stationäre Behandlung, Notfallversorgung und Rehabilitation ermittelt. Die Differenz der Arzneimittelmehr- und der Behandlungskosten eines KV-Ereignisses geteilt durch 12 ergibt die Einsparung pro Patient und Jahr.

Ergebnisse. Bei 12 mit Degarelix behandelten PCa-Patienten mit KV-Ereignissen in der Vorgeschichte entstehen im Vergleich zu
Leuprorelin zusätzliche Arzneimittelkosten von $3111 €$ pro Jahr. Für ein KV-Ereignis werden Kosten von $8447 €$ pro Jahr vermieden. Hieraus beträgt die Einsparung $445 €$ pro Patient und Jahr für jeden mit Degarelix behandelten Patienten.

Schlussfolgerung. Degarelix ist im Vergleich zu Leuprorelin für PCa-Patienten mit KV-Ereignissen in der Vorgeschichte kosteneffektiv.

\section{Schlüsselwörter}

Prostatakarzinom - Androgendeprivationstherapie $\cdot$ Kosteneffektivität · „Number needed to treat" . Degarelix

\section{Cost effectiveness of GnRH antagonists in patients with prostate cancer and cardiovascular risk. Comparative analysis against Leuprorelin by the Number Needed to Treat}

\section{Abstract}

Background. Recent studies suggest that androgen deprivation therapy (ADT) is associated with increased cardiovascular (CV) risk for patients with hormone-sensitive prostate cancer ( $\mathrm{PCa}$ ) and pre-existing CV disease. This risk seems to be different for the gonadotropin-releasing hormone $(\mathrm{GnRH})$ agonists leuprolide and goserelin and $\mathrm{GnRH}$ antagonists, whereas the slightly more expensive $\mathrm{GnRH}$ antagonist shows a beneficial risk profile. The present study assesses the cost effectiveness of degarelix compared to leuprolide for PCa patients with increased CV risk.

Methods. This analysis is based on a pooled analysis of six phase III, randomized, controlled trials comparing the $\mathrm{GnRH}$ agonists leuprolide and goserelin with the GnRH antagonist degarelix. For the combined endpoint of $\mathrm{CV}$ events or death a superiority of degarelix was determined with a Number-Needed-toTreat of 12. From the perspective of German statutory health insurance, this evaluation estimates and compares the additional drug costs of degarelix treatment to the cost of one (avoided) CV event. The CV event costs were estimated via emergency treatment and transportation, inpatient treatment, and rehabilitation. The difference of these two cost pools divided by 12 yields the average saving per patient and year.

Results. For every 12 PCa patients with CV history that are treated with $\mathrm{GnRH}$ antagonists to prevent one $\mathrm{CV}$ event, there will be additional drug costs in comparison with leuprolide treatment of $€ 3111$ per year. Costs of $€ 8447$ per year are prevented. Therefore, each patient with a history of $\mathrm{CV}$ who is treated with degarelix instead of a leuprolide generates savings of $€ 445$ per patient and year.

Conclusions. Compared to leuprolide, degarelix is cost effective for patients with increased $\mathrm{CV}$ risk.

Keywords

Prostate cancer - Androgen deprivation therapy · Cost-effectiveness analysis · Numbers needed to treat $\cdot$ Degarelix hier betrachteten KV-Ereignissen kann grundsätzlich davon ausgegangen werden, dass ein schnelles medizinisches Eingreifen notwendig ist. Dabei wird in aller Regel der Betroffene mittels Rettungsfahrt ( $\$ 5)$ bzw. Krankentransport (\$6) in das nächstmögliche Krankenhaus gebracht.

Aus $₫ 5$ (3) SGB V geht hervor, dass Rettungswagen (RTW), Notarztwagen (NAW) oder Notarzteinsatzfahrzeug (NEF) bei Patienten verordnet werden können, „bei denen vor oder während des Transportes lebensrettende Sofortmaßnahmen durchzuführen oder $\mathrm{zu}$ erwarten sind, für die ein Notarzt erforderlich ist“. Aus Veröffentlichungen der AOK NordWest geht hervor, dass sowohl RTW und NEF bei KV-Ereignissen verordnet werden, da der Notarzt üblicherweise nicht gemeinschaftlich mit dem Rettungswagen ausrückt [25]. Da sowohl argumentiert werden kann, dass nicht bei allen KV-Ereignissen lebensret- tende Sofortmaßnahmen und damit ein Notarzt notwendig ist, auf der anderen Seite aber auch bei Ereignissen dieser Art ein teurerer Rettungshubschrauber zum Einsatz kommen kann, wird hier angenommen, dass sowohl RTW als auch NEF zum Einsatz kommen.

Um die Kosten für die Notfallversorgung durch Krankentransporte mit notärztlicher Begleitungserfordernis $\mathrm{zu}$ ermitteln, wurden regionale Vergütungsvereinbarungen herangezogen, da 


\section{Zusätzlich anfallende \\ GKV-Arzneimittelkosten pro Jahr}

\section{KV-Ereignis- verursachte jährliche GKV-Kosten}

Abb. 1 ॥ Struktur der ökonomischen Analyse

die Vergütungen zwischen Leistungserbringern und Kostenträgern regional individuell vereinbart werden. Durch eine Freihandsuche im Internet wurden Vereinbarungen aus unterschiedlichen Regionen ermittelt. Da die Vergütungen zwischen städtischem und ländlichem Raum differieren, wurden aus den identifizierten Vergütungsvereinbarungen Entgeltmittelwerte für RTW und NEF gebildet [5, 9, 14, 19, 26, 28, 29]. Diese Mittelwerte wurden dann zu den Gesamtkosten für die Notfallversorgung summiert.

Die Kosten für die Krankenhausbehandlung von KV-Ereignissen wurden anhand von ICD-Schlüsseln operationalisiert (ICD-10-2014, • Tab. 3), als Hauptdiagnose genutzt und per DRG-Browser des InEK (Datenjahr 2014) abgefragt. Es wurden aus Gründen der Repräsentativität nur solche DRG eingeschlossen, die innerhalb der jeweiligen Hauptdiagnose eine Fallzahl von mindestens 100 Patienten aufwiesen.

Da die Vergütung der Krankenhäuser auf einer Fallzahldatenbasis und einem zwischen dem GKV-Spitzenverband und der Deutschen Krankenhausgesellschaft vereinbarten Bundesbasisfallwert (BBFW) aus unterschiedlichen Jahren basiert, wurden die Fallkosten anhand des BBFW für 2016 (3311,98 €) und dem DRG-Katalog 2014 bestimmt [13]. Um einen Kostenpunkt für alle betrachteten Endpunkte zu ermitteln, wurde eine Gewichtung nach Fallzahlen vorgenommen.

Die Tagessätze für eine rehabilitative Maßnahme wurden anhand eines „Gutachtens zur aktuellen und perspektivischen Situation der Einrichtungen im Bereich der medizinischen Rehabilitation“ der GEBERA ermittelt. Für das Gutachten wurden verschiedene Rehabilitationseinrichtungen ( $n=187)$ nach den Vergütungen für Heilverfahren,
Anschlussheilbehandlungen und ambulanter Rehabilitationen im Rahmen der GKV befragt [4].

Eine Einschränkung bei der Rehabilitationsleistung auf Patienten im Rentenbezug - also über 65 Jahren - wurde vorgenommen, da der Erkrankungsgipfel bei Patienten mit PCa laut dem Zentrum für Krebsregisterdaten (ZfKD) und dem Robert Koch-Institut (RKI) über 65 Jahren liegt und die GKV für Rehabilitationsleistungen nur bei Patienten im Rentenbezug der Kostenträger ist. Die Kosten für eine kardiologische Rehabilitation, die nach KV-Ereignissen üblich ist, sind für Patienten im Rentenbezug, die rehabilitationswillig und -fähig sind, von der GKV zu tragen. Gemäß der ZfKD und RKI-Daten kann davon ausgegangen werden, dass PCa-Patienten in der Mehrheit $(75,41 \%)$ das 65 . Lebensjahr erreicht haben bzw. bereits im Rentenbezug sind [23].

Die Tagessätze neurologischer Rehabilitationen nach Schlaganfall sind möglicherweise höher, waren aber nicht valide zu ermitteln. Daher wurden ausschließlich Kosten für eine kardiologische Rehabilitation angewendet.

\section{Sensitivitätsanalysen}

Zur Sicherung der Ergebnisse wurden folgende Sensitivitätsanalysen vorgenommen: (1) Vergleich mit dem günstigsten auf dem Markt befindlichen Leuprorelin-Präparat, (2) Ermittlung der stationären KV-Ereigniskosten aus einer GKV-Routinedatenanalyse.

\section{Ergebnisse}

Die Ermittlung der NNT aus der Arbeit von Albertsen et al. ergab über alle sechs Studien 12,2 (95\%-KI 7,6-30,8). Gerundet ergibt sich eine NNT von 12 Patienten, welche mit GnRH-Antagonisten im
Vergleich zu den GnRH-Agonisten Leuprorelin und Goserelin behandelt werden müssen, um ein KV-Ereignis oder Tod zu verhindern.

\section{Therapieinduzierte Arzneimittel- kosten}

Die Mehrkosten im Rahmen der Dauertherapie, welche der GKV durch die Behandlung mit Degarelix im Vergleich zu einer Behandlung mit GnRH-Agonisten (hier Leuprorelin) entstehen, wurden anhand der Präparate Firmagon ${ }^{\circledR}$ $80 \mathrm{mg}$, Trenantone ${ }^{\circledR} 11,25 \mathrm{mg}$ (als verordnungsstärkstes Leuprorelin-Präparat, $34,5 \%$ der Verordnungen in 10/13-09/14 [16]) und Leuprone ${ }^{\circledR}$ HEXAL 5 mg (als Sensitivitätsanalyse) ermittelt.

Aus der Fachinformation zu Degarelix geht eine 2-mal jährliche Überprüfung des Testosteron-Wertes (Gebührenordnungsposition [GOP] 32.358 à $5 €$ ) des aktuellen einheitlichen Bewertungsmaßstabs (EBM, 2. Quartal 2016) und des PSA-Wertes (GOP 32.351 à 4,80€) hervor [10]. Beider Anwendung von Leuprorelin-Präparaten ist laut Fachinformation der Testosteron-Wert 4-mal zu überprüfen. Außerdem sind eine initiale Antiandrogengabe und die 2-malige Überprüfung der sauren Phosphatase (GOP 32.079 à $0,25 €)$ erforderlich $([15,27]$; - Tab.4). Hieraus ergeben sich die Jahrestherapiekosten in der Dauertherapie aus Sicht der GKV pro Patient (Anzahl Packungen pro Jahr $x$ GKV-Kosten pro $\mathrm{Pa}$ ckung + Kosten für zusätzlich notwendige GKV-Leistungen; • Tab. 5). Beim Vergleich von Firmagon ${ }^{\circledR}$ mit Trenantone ${ }^{\circledR}$ beträgt die Kostendifferenz pro Patient $259,28 €$. Im Vergleich zum günstigsten Leuprorelin-Präparat beträgt die Differenz $629,18 €$.

\section{Behandlungskosten kardiovasku- lärer Ereignisse}

Als Kosten für die Notfallversorgung ergab die Recherche von Entgeltvereinbarungen, eine Spannweite von 336,61738,00 € für ein NEF und 235,00-932,23 € für ein RTW. Die Mittelwerte der Entgeltvereinbarungen betragen für ein NEF $474,36 €$ und für ein RTW $491,06 €$. Somit beträgt die Summe der Mittelwerte 
Hier steht eine Anzeige. Springer für ein NEF und ein RTW 965,42 €, welche als Kosten für die Notfallversorgung angenommen wurden.

Die Kosten für die Krankenhausbehandlung bei einem KV-Ereignis, ermittelt durch eine Gewichtung der Fallzahlen, betragen über alle relevanten Krankenhausfälle 5739,40€ (• Tab. 6).

$\mathrm{Zu}$ den Kosten der Rehabilitation heißt es im Gutachten der GEBERA: „Die aktuellen Tagessätze [2010] in der Kardiologie bewegen sich zwischen $90 €$ und $123 €$, wobei die Mediane je nach Rehabilitationsträger zwischen $110 €$ und $114 €$ betragen“ [7].

Die Tagessätze einzelner Einrichtungen erwiesen sich als schwieriger $\mathrm{zu}$ recherchieren, da diese individuell zwischen den Einrichtungen und Kostenträgern verhandelt werden. Durch eine Freihandrecherche konnte zusätzlich der Tagessatz einer Einrichtung recherchiert werden, der sich mit den Ergebnissen des Gutachtens deckt.

Es wurde mit dem Mindestmedian von $110 €$ pro Tag aus der GEBERAUmfrage weiter gearbeitet. Daraus ergeben sich bei einer Rehabilitationsdauer von mindestens 3 Wochen Rehabilitationskosten in Höhe von $2310 €$. Wird hierauf die Quote der PCa-Patienten im Rentenbezug angewendet (75,41\%), ergeben sich pro Patient Kosten in Höhe von $1741,97 €$ für eine kardiologische Rehabilitation.

Insgesamt wurden somit KV-Ereignis-induzierte Gesamtkosten für die Notfallversorgung, die Behandlung im Krankenhaus und für die Rehabilitation in Höhe von $8446,79 €$ in die Kalkulation einbezogen (•Tab. 7).

\section{Gegenüberstellung}

Bei 12 mit Degarelix behandelten PCaPatienten mit KV-Vorgeschichte entstehen im Vergleich zu Trenantone ${ }^{\circledR}$ zusätzliche Arzneimittelkosten in Höhe von $3111,36 €$. Demgegenüber würden durchschnittliche Kosten für ein KVEreignis in Höhe von $8446,79 €$ verhindert. Somit besteht eine Mehrausgabe in Höhe von 5335,43€ für 12 Patienten bei der Behandlung mit Trenantone ${ }^{\circledR}$. Dies entspricht im Basisfall einer jährlichen Einsparung in Höhe von 444,62€ pro PCa-Patient mit KV-Vorgeschichte, wenn er mit Degarelix statt Leuprorelin behandelt würde (• Tab. 8).

\section{Sensitivitätsanalysen}

Bei der Behandlung von 12 Patienten mit Degarelix anstelle von Leuprone ${ }^{\circledR}$ HEXAL ergeben sich 7550,16€ Arzneimittelmehrkosten. Bei gleichbleibenden Kosten für ein $\mathrm{KV}$-Ereignis in Höhe von $8446,79 €$ ergibt sich eine Einsparung pro Patient von $74,72 €$, wenn alle 12 Patienten mit Degarelix behandelt werden.

Bezieht man die Ergebnisse einer GKV-Routinedatenanalyse zu den tatsächlichen Kosten eines Herzinfarktes $(9875 €)$ bzw. Schlaganfalls (9366€) mit ein, ergeben sich fallzahlgewichtete durchschnittliche Krankenhauskosten von $6850,11 €$ und Gesamtkosten für ein KV-Ereignis von 9557,50€ [24]. Hieraus ergeben sich gegenüber der Leitsubstanz Trenantone ${ }^{\circledR}$ Einsparungen von 537,18€ pro Patient und Jahr (NNT 12; 3111,36€ NNT-induzierte Arzneimittelmehrkosten).

\section{Diskussion}

Mit dieser Studie liegt der erste Vergleich zur Kosteneffektivität verschiedener ADT-Präparate für die Patientengruppe mit fPCa und einer kardiovaskulären Vorgeschichte vor. Die NNT hat sich in der Medizin und Gesundheitsökonomie als ein Instrument etabliert, um die Effektivität einer Therapie besser einzuschätzen und begreifbar zu machen. So kann der „budget impact“ für die GKV verschiedener Therapien mit einer Kennzahl dargestellt werden, die für alle Akteure im Gesundheitswesen (Kostenträger, Ärzte und mit Einschränkungen auch Patienten) allgemeinverständlich ist. Das heißt anhand der NNT können therapieinduzierte Mehrkosten oder Einsparungen abgebildet werden. Eine gute Übersicht an Praxisbeispielen bietet das systematische Review von Moore, McQuay oder das arznei-telegramm [1, 21].

Die Mehrkosten für eine Therapie mit Degarelix werden durch die verhinderten Kosten für KV-Ereignisse auch unter Berücksichtigung von Sensitivitätsanalysen 
Tab. 4 Kosten zusätzlich notwendige GKV-Leistungen

\begin{tabular}{|c|c|c|}
\hline Präparat $[10,15,27]$ & zGKV-L & $€ / J a h r$ \\
\hline Degarelix & Keine $^{a}$ & 0,00 \\
\hline \multirow[t]{4}{*}{ Leuprorelin } & $2 \times$ Testosteron (GOP 32.358) & 10,00 \\
\hline & $2 \times$ Saure Phosphatase (GOP 32.079) & 0,50 \\
\hline & $90 \times$ Antiandrogengabe (à $0,32 €^{b}$ ) & 28,80 \\
\hline & Summe & 39,30 \\
\hline \multicolumn{3}{|c|}{$\begin{array}{l}\text { 'Keine, die bei Leuprorelin nicht auch anfallen, daher hier nicht weiter berücksichtigt (z. B. Kosten } \\
\text { ärztlicher Versorgung im Rahmen der Versichertenpauschale, die auch die Arzneimittelinjektionen } \\
\text { abdeckt) } \\
\text { '4 Wochen 3-mal täglich eine Tbl. Flutamid } 250 \mathrm{mg} \text {, z. B. Flutamid } 250 \mathrm{mg} \text {, PZN } 07689105 \text {, N3 } \\
\text { à } 84 \text { Tbl., (AVP-Rabatte }=26,86 € / 84 \text { Tbl.) }\end{array}$} \\
\hline
\end{tabular}

Tab. 5 Arzneimittelkosten pro Patient

\begin{tabular}{|c|c|c|c|}
\hline Präparat & $\begin{array}{l}\text { GKV AM-Kosten/Packung } \\
(\epsilon)\end{array}$ & $\begin{array}{l}\text { ZGKV-L } \\
(\epsilon)\end{array}$ & $\begin{array}{l}\text { JTK } \\
(\epsilon)\end{array}$ \\
\hline Firmagon ${ }^{\circledR} 80 \mathrm{mg}^{\mathrm{a}}$ & 514,40 & 0,00 & 2057,60 \\
\hline Trenantone ${ }^{\circledR} 11,25 \mathrm{mg}$ & 879,51 & 39,30 & 1798,32 \\
\hline Leuprone ${ }^{\circledR}$ HEXAL 5 mg & 694,56 & 39,30 & 1428,42 \\
\hline \multicolumn{4}{|c|}{$\begin{array}{l}\text { Quelle: Lauer Taxe mit Preis- und Produktstand } 15.07 .2016 \\
\text { GKV AM-Kosten Apothekenverkaufspreis (AVP) - Herstellerrabatt It. §130a SGB V - Apotheken- } \\
\text { abschlag von 1,77 €, zGKV-L zusätzlich notwendige GKV-Leistungen gemäß Fachinformation, JTK } \\
\text { Jahrestherapiekosten } \\
\text { aBetrachtung der Dauertherapie, daher Starterpackung nicht berücksichtigt }\end{array}$} \\
\hline
\end{tabular}

Tab. 6 Stationäre Kosten - relevante DRG

\begin{tabular}{|c|c|}
\hline KV-Ereignisse [2] & $\begin{array}{l}\text { - DRG mit FZ } \geq 100[17,18] \\
\text { - Fallzahl (FZ) } \\
\text { - Mittelwert (MW, FZ-gewichtet) }\end{array}$ \\
\hline $\begin{array}{l}\text { Arterielle Embolien } \\
\text { und thrombot. Ereig- } \\
\text { nisse }\end{array}$ & $\begin{array}{l}\text { - F08B, F08D-E, F14B, F28A, F59A-D, F65B; } \\
\text { - FZ } 3888 \\
\text { - MW 6520,79€ }\end{array}$ \\
\hline $\begin{array}{l}\text { Hämorrhag., zerebro- } \\
\text { vask. Zustände }\end{array}$ & $\begin{aligned} & \text { 901D, A09C, A11D, A13D, B02C-D, B20B, B20E-F, B36B, B39B, B70A-F, } \\
& \text { B70H-I } \\
- & \text { FZ 11.248 } \\
- & \text { MW } 8752,38 €\end{aligned}$ \\
\hline $\begin{array}{l}\text { Ischäm., zerebrovask. } \\
\text { Zustände }\end{array}$ & $\begin{aligned}- & \text { B02C, B04A-D, B12Z, B20B, B20E, B39A-C, B42A-B, B44A-D, B69D, } \\
& \text { B70A-I } \\
- & \text { FZ 49.864 } \\
- & \text { MW 5437,44€ }\end{aligned}$ \\
\hline Myokardinfarkt & $\begin{array}{l}- \text { A11F, A13E-F, F06D, F06F, F07B, F09B, F12D, F15Z, F24A-B, F36B, } \\
\text { F41B, F43C, F48Z, F49D, F52A-B, F56A, F58A, F60A-B } \\
\text { - FZ 34.567 } \\
\text { - } \text { MW 5998,72€ }\end{array}$ \\
\hline $\begin{array}{l}\text { Andere ischäm. Herz- } \\
\text { erkrankung }\end{array}$ & $\begin{array}{l}\text { - F01C, F01F-G, F03E, F06C, F06E-F, F07B, F24B, F36B, F49D, F49F-G, } \\
\text { F56B, F58A-B, F66A-B, F75D } \\
\text { - FZ } 27.289 \\
- \text { MW } 4609,08 €\end{array}$ \\
\hline
\end{tabular}

überkompensiert. Durch eine zielgerichtete Therapieauswahl für PCa-Patienten, welche vor Einleitung einer medikamentösen ADT ein KV-Ereignis erlitten haben, könnte die GKV durchschnittliche Kosten zwischen 74,72-537,18 € pro Patient einsparen. Dies bedeutet, dass die bieten. Neben der positiven Bilanz zur Kosteneffektivität kommen aus Sicht der Patienten bei der Vermeidung von kardiovaskulären Ereignissen auch Faktoren wie gewonnene Lebensqualität hinzu.

Durch die in der vorliegenden Studie gezeigten Sensitivitätsanalysen ist die Kosteneffektivität für eine Degarelix-Therapie für $\mathrm{PCa}$-Patienten mit einer Vorgeschichte an KV-Ereignissen auch unter geänderten Annahmen bestätigt, womit ein Argument gegen Verordnungseinschränkungen gegenüber Kassenärztlichen Vereinigungen gegeben ist.

Limitationen der Studie sind der kombinierte Endpunkt aus kardiovaskulären Ereignissen oder Tod und die daraus resultierende Berechnung der NNT auf Basis einer gepoolten Post-hoc-Analyse von KV-Ereignissen. Demnach sind weitere gesundheitsökonomische Evaluierungen erforderlich, welche auf klinischen Studien mit einem direkten Vergleich von KV-Ereignissen bei GnRH-Agonisten und GnRH-Antagonisten basieren. Die Ergebnisse der PRONOUNCE-Studie [31], welche die kardiovaskuläre Sicherheit von Degarelix im Vergleich zu Leuprorelin untersucht, können für eine Validierung und Aktualisierung der Ergebnisse aus der vorliegenden Evaluation herangezogen werden.

Außerdem sind weitere Studien zu den tatsächlich entstehenden Kosten geplant, um den in dieser Studie betrachteten Sachverhalt in der Behandlungsrealität $\mathrm{zu}$ untersuchen. Dies wäre z. B. in Kooperation mit einer Krankenkasse möglich.

\section{Fazit für die Praxis}

- Degarelix ist im Vergleich zu Leuprorelin für fPCa-Patienten mit einer kardiovaskulären Vorgeschichte kosteneffektiv.

- Die präparatbezogenen Mehrkosten bei einer Therapie mit Degarelix im Vergleich zu einer Therapie mit Leuprorelin werden für fPCa-Patienten mit einer kardiovaskulären Vorgeschichte durch vermiedene Kosten für ein KV-Ereignis um durchschnittlich $444,62 €$ pro Patient überkompensiert. 
Tab. 7 KV-Ereignis-induzierte Gesam-

t-GKV-Kosten

Kostenposition

Betrag

(є)

Krankenhaus (fallzahlgewichte- $\quad 5739,40$ ter Mittelwert)

Krankentransport [12, 19, 26]

965,42

21-Tage-Rehabilitation [7, 22]

1741,97

GKV-Kosten gesamt

8446,79

- In den Sensitivitätsanalysen wurde gezeigt, dass Degarelix auch bei einem Vergleich der Therapiekosten mit dem günstigsten und nicht dem verordnungsstärksten Leuprorelin-Präparat, oder wenn Kosten für Herzinfarkt und Apoplex basierend auf GKV-Routinedaten herangezogen werden, kosteneffektiv ist (durchschnittliche Einsparungen von $74,72 €$ pro Patient, bzw. 537,18 € pro Patient).

- Kostenaufwand und Kosteneinsparungen entstehen bei der Behandlung von $\mathrm{PPCa}$-Patienten mit einem erhöhten Risiko für kardiovaskuläre Ereignisse in unterschiedlichen Sektoren. Hierin könnte ein Ansatz zur integrierten Versorgung der untersuchten Patientengruppe liegen.

\section{Korrespondenzadresse}

\section{Anderson}

Ecker + Ecker GmbH

Warburgstraße 50, 20354 Hamburg,

Deutschland

d.anderson@gmx.de

\section{Einhaltung ethischer Richtlinien}

Interessenkonflikt. J. Lehmann erhielt in den vergangenen Jahren Honorare für Beratungstätigkeiten, Vorträge, Reisekostenübernahmen und Studienunterstützungen von den Unternehmen Janssen, Takeda, Ferring Arzneimittel, Sanofi-Aventis, Amgen, Apogepha. Für die wissenschaftliche Begleitung der vorliegenden Studie erhielt er kein Honorar.

Die Studie wurde unterstützt von der Ferring Arzneimittel GmbH, Kiel. Dr. V. Donatz und S. Vosgerau sind Angestellte der Ferring Arzneimittel $\mathrm{GmbH}$.

Ecker + Ecker, Hamburg, hat die Studie im Auftrag der Ferring Arzneimittel GmbH durchgeführt. D. Anderson ist Angestellte bei Ecker + Ecker und Dr. T. Ecker ist Geschäftsführer von Ecker + Ecker.

\begin{tabular}{|c|c|c|c|c|}
\hline Analyse & Komparator & $\begin{array}{l}\text { Verhinderte } \\
\text { Kosten } \\
\text { (I) } \\
\text { (€) }\end{array}$ & $\begin{array}{l}\text { Arzneimittel- } \\
\text { mehrkosten } \\
\text { (II) } \\
\text { (€) }\end{array}$ & $\begin{array}{l}\text { Einsparungen } \\
\text { pro Patient/Jahr } \\
\text { (I-II/NNT) } \\
\text { (€) }\end{array}$ \\
\hline Basisfall & Trenantone ${ }^{\circledR}$ & 8446,79 & 3111,36 & 444,62 \\
\hline $\begin{array}{l}\text { Sensitivitätsanalyse } \\
\text { mit günstigstem } \\
\text { ADT-Produkt als } \\
\text { Komparator }\end{array}$ & $\begin{array}{l}\text { Leuprone }{ }^{\circledR} \mathrm{HE}- \\
\text { XAL }\end{array}$ & 8446,79 & 7550,16 & 74,72 \\
\hline $\begin{array}{l}\text { Sensitivitätsanalyse: } \\
\text { KV-Ereigniskosten } \\
\text { basierend auf } \\
\text { GKV-Routinedaten }\end{array}$ & Trenantone ${ }^{\circledR}$ & 9557,50 & 3111,36 & 537,18 \\
\hline
\end{tabular}

Dieser Beitrag beinhaltet keine von den Autoren durchgeführten Studien an Menschen oder Tieren.

Open Access. Dieser Artikel wird unter der Creative Commons Namensnennung 4.0 International Lizenz (http://creativecommons.org/licenses/by/4.0/deed. de) veröffentlicht, welche die Nutzung, Vervielfältigung, Bearbeitung, Verbreitung und Wiedergabe in jeglichem Medium und Format erlaubt, sofern Sie den/die ursprünglichen Autor(en) und die Quelle ordnungsgemäßnennen, einen Linkzur Creative Commons Lizenz beifügen und angeben, ob Änderungen vorgenommen wurden.

\section{Literatur}

1. A.T.I. Arzneimittelinformation Berlin (1998) Number needed to Treat - Eine Hilfe für Therapieentscheidungen. Arznei Telegr 5/98:47-50

2. Albertsen PC, Klotz L, Tombal B et al (2014) Cardiovascular morbidity associated with gonadotropin releasing hormone agonists and an antagonist. Eur Urol 65:565-573

3. Amelung VE, Mühlbacher A, Krauth C, Gabler Wirtschaftslexikon (2017) Stichwort: Gesundheitsökonomische Evaluation. http:// wirtschaftslexikon.gabler.de/Archiv/17874/ gesundheitsoekonomische-evaluation-v10.html. Zugegriffen: 14.10.2016

4. Augurzky B, Reichert AR, Scheuer M (2011) Faktenbuch Medizinische Rehabilitation 2011

5. Bayerisches Rotes Kreuz Kreisverband Miesbach (2016) Der Rettungsdienst in Bayern. https://kvmiesbach.brk.de/rettungsdienst/derrettungsdienst-in-bayern. Zugegriffen: 16. Aug 2016

6. Bekelman JE, Mitra N, Handorf EA et al (2015) Effectiveness of androgen-deprivation therapy and radiotherapy for older men with locally advanced prostate cancer.J Clin Oncol 33(7):716-722. doi:10. 1200/jco.2014.57.2743

7. Borges P, Zimolong A (2009) Gutachten zur aktuellen und perspektivischen Situation der Einrichtungen im Bereich der medizinischen Rehabilitation - Aktualisierung 2009. http:// www.sucht.de/tl_files/pdf/gutachten/1-151024 aktiva\%20GA\%20Reha_2016_final.pdf.Zugegriffen: 16 . Aug 2016

8. Deutsches Institut für Medizinische Dokumentation und Information (DIMDI) (2014) ICD-10 Katalog. http://www.dimdi.de/static/de/klassi/ icd-10-gm/index.htm. Zugegriffen: 16 . Aug 2016
9. Ennepe-Ruhr-Kreis (2015) Gebührensatzung für den Krankentransport-und Rettungsdienstdes Ennepe-Ruhr-Kreises vom 23. Mai 2015. http://www. witten.de/fileadmin/user_upload/Dokumente/ sta10/orecht/or3/314.pdf. Zugegriffen: 16. Aug 2016

10. Ferring Arzneimittel (2014) Fachinformation Degarelix Fachinformation. Stand:Oktober. http:// www.fachinfo.de.Zugegriffen: 16. Aug 2016

11. Garg V, Shen X, Cheng Y et al (2013) Use of number needed to treat in cost-effectiveness analyses. Ann Pharmacother 47:380-387

12. Gemeinsamer Bundesausschuss (2005) Richtlinien über die Verordnung von Krankenfahrten und Krankentransportleistungen. https://www.g-ba de/downloads/62-492-74/RL-Khtransport-200412-21.pdf. Zugegriffen: 16. Aug 2016

13. GKV Spitzenverband, Deutsche Krankenhausgesellschaft (2015) Vereinbarung gemäß $§ 10$ Abs. 9 KHEntgG für den Vereinbarungszeitraum 2016. https://www.gkv-spitzenverband. de/media/dokumente/krankenversicherung_ 1/krankenhaeuser/budgetverhandlungen/ bundesbasisfallwert/KH_BBFW_2016.pdf. Zugegriffen: 16. Aug 2016

14. Hansestadt Herford (2015) Gebührensatzung für den Krankentransport und die Notfallrettung in der Hansestadt Herford. http://www.herford.de/ media/custom/1050_1191_1.PDF?1450708247. Zugegriffen: 16. Aug 2016

15. Hexal AG (2014) Leuprorelin Hexal 5 mg; Fachinformation. Stand: Juli 2014. http://www.fachinfo.de. Zugegriffen: 16. Aug 2016

16. IMS Health (2014) DPM - L02 Market-MAT 9-2014 (Data on file)

17. Institut für das Entgeltsystem im Krankenhaus (InEK) (2014) DRG-Katalog. http://www.gdrg.de/cms/inek_site_de/layout/set/einspaltig/ G-DRG-System_2016/Fallpauschalen-Katalog/ Fallpauschalen-Katalog_2016. Zugegriffen: 16. Aug 2016

18. Institut Für Das Entgeltsystem Im Krankenhaus (InEK) (2014) G-DRG-Browser 2012/2014. http://www.g-drg.de/inek_site_de/layout/ set/einspaltig/Archiv/DRG_Systemjahr_2014_ Datenjahr_2012.Zugegriffen:14.10.2016

19. Kreis Nordfriesland (2012) Entgeltvereinbarung Rettungsdienst Kreis Nordfriesland. https:// www.nordfriesland.de/media/custom/2271_73_ 1.PDF?1361635707.Zugegriffen: 16. Aug 2016

20. Merseburger AS, Sedding D, Hüter K (2015) Kardiovaskuläre Risikopatienten unter Androgenentzugstherapie. Urologe 55(2):218-225. doi:10. 1007/s00120-015-0013-1 
21. Moore A, McQuay HJ (2008) Was ist die Number Needed to Treat (NNT)? ZAllg Med 84:161-164

22. Rehaklinik Bad Salzelmen (2010) Rehaklinik Bad Salzelmen, Häufig gestellte Fragen zur Rehabilitation. http://www.rehaklinik-bad-salzelmen.de/ bibliothek/Reha_Bad_Salzelmen/pdf/Haeufig_ gestellte_Fragen_zur_Rehabilitation.pdf. Zugegriffen: 16. Aug 2016

23. Robert Koch-Institut (RKI) (2015) Krebsregisterdaten.www.krebsdaten.de/abfrage.Zugegriffen: 16 Aug 2016

24. Rüssel C, Guthoff-Hagen S, Donatz V (2015) Androgen Deprivation Therapy (ADT) and cardiovascular risk (data on file). JClin Oncol 33(Suppl 7):Abstr 219

25. Simolka F (2011) Können wir uns das alles noch leisten? "Herzinfarkt erkennen und richtig handeln" (HEUH) - Wenn das Leben aus dem Takt gerät. Infoveranstaltung am Samstag, 16. Juli, Paderborn.

26. Stadt Osnabrück (2014) Entgeltvereinbarung 2014 Rettungsdienst Stadt Osnabrück. http:// www.osnabrueck.de/fileadmin/eigene_Dateien/ Entgeltevereinbarung_Osnabrueck 2014 rettungsdienst.pdf.Zugegriffen: 16 . Aug 2016

27. Takeda (2013) Trenantone $11,25 \mathrm{mg}$; Fachinformation. Stand: November 2013. http://www.fachinfo. de. Zugegriffen: 16 . Aug 2016

28. vdek - Verband der Ersatzkassen (2016) Rettungsdienst Berlin - Aktuelle Rettungsdienstgebühren. https://www.vdek.com/LVen/BERBRA/ Vertragspartner/Rettungsdienst/Berlin.html. Zugegriffen: 16. Aug 2016

29. vdek - Verband der Ersatzkassen (2016) Rettungsdienst Brandenburg - Rettungsdienstgebühren. https://www.vdek.com/LVen/BERBRA/ Vertragspartner/Rettungsdienst/Brandenburg. html.Zugegriffen: 16. Aug 2016

30. Ziehr DR, Chen MH, Zhang D, Braccioforte MH et al (2014) Association of androgen-deprivation therapy with excess cardiac-specific mortality in men with prostate cancer. BJU Int 116(3):358-365. doi:10.1111/bju.12905

31. Ferring Pharmaceuticals (2017) A trial comparing cardiovascular safety of degarelix versus leuprolide in patients with advanced prostate cancer and cardiovascular disease (PRONOUNCE), clinicaltrials.govidentifier:NCT02663908. https://clinicaltrials.gov/ct2/show/NCT02663908. Zugegriffen: 14.10.2016

\section{Erratum}

Urologe $2017 \cdot 56: 924$

DOI 10.1007/s00120-017-0426-0

Online publiziert: 19. Juni 2017

๑) Springer Medizin Verlag GmbH 2017. Dieser Artikel ist eine Open-Access-Publikation.

CrossMark

\section{Anderson ${ }^{1} \cdot$ J. Lehmann ${ }^{2} \cdot$ T. Ecker ${ }^{1} \cdot$ S. Vosgerau ${ }^{3} \cdot$ V. Donatz}

'Ecker + Ecker GmbH, Hamburg, Deutschland

${ }^{2}$ Klinik für Urologie, Städtisches Krankenhaus, Kiel, Deutschland

${ }^{3}$ Ferring Arzneimittel $\mathrm{GmbH}$, Kiel, Deutschland

\section{Erratum zu: Kosteneffektivität von GnRH-Antagonisten bei Patienten mit Prostatakarzinom und kardiovaskulärem Risiko. Vergleichende Analyse gegenüber Leuprorelin anhand der Number Needed to Treat}

\section{Erratum zu: \\ Der Urologe 2017 \\ DOI 10.1007/s00120-017-0382-8}

Leider ist in diesem Beitrag ein Fehler im englischen Titel aufgetreten. Wir bitten den korrekten Titel "Cost-effectiveness of $\mathrm{GnRH}$ antagonists in patients with prostate cancer and cardiovascular risk. Comparative analysis with leuprolide on the basis of the Number Needed to Treat" zu beachten und den Fehler zu entschuldigen.

\section{Korrespondenzadresse}

\section{Anderson}

Ecker + Ecker GmbH

Warburgstraße 50, 20354 Hamburg

Deutschland

d.anderson@gmx.de 